\title{
The Swiss joint information platform for natural hazards
}

\author{
Philipp Angehrn a, *, Sabina Steiner ${ }^{a}$, Christophe Lienert ${ }^{b}$ \\ ${ }^{a}$ GIN Secretariat, clo Federal Office for the Environment, Switzerland, philipp.angehrn@bafu.admin.ch, \\ sabina.steiner@bafu.admin.ch \\ ${ }^{b}$ Canton Aargau, Department Construction, Traffic and Environment, Switzerland, christophe.lienert@ag.ch \\ * Corresponding author
}

Keywords: real-time cartography, interactive maps, decision support, natural hazards, risk management

\section{Abstract:}

The Swiss Joint Information Platform for Natural Hazards (GIN) has been realized from 2008 to 2010 as part of the Swiss federal government's OWARNA project, which aimed at optimizing warning and alerting procedures against natural hazard. The first online-version of the platform went productive in 2011 with the primary goal of providing measured and forecast natural hazard data in form of processed cartographic, graphic and other multimedia products to professional users - before, during and after natural hazard events. In Switzerland water-, weather-, snow- and earthquake-related hazards are the most relevant ones.

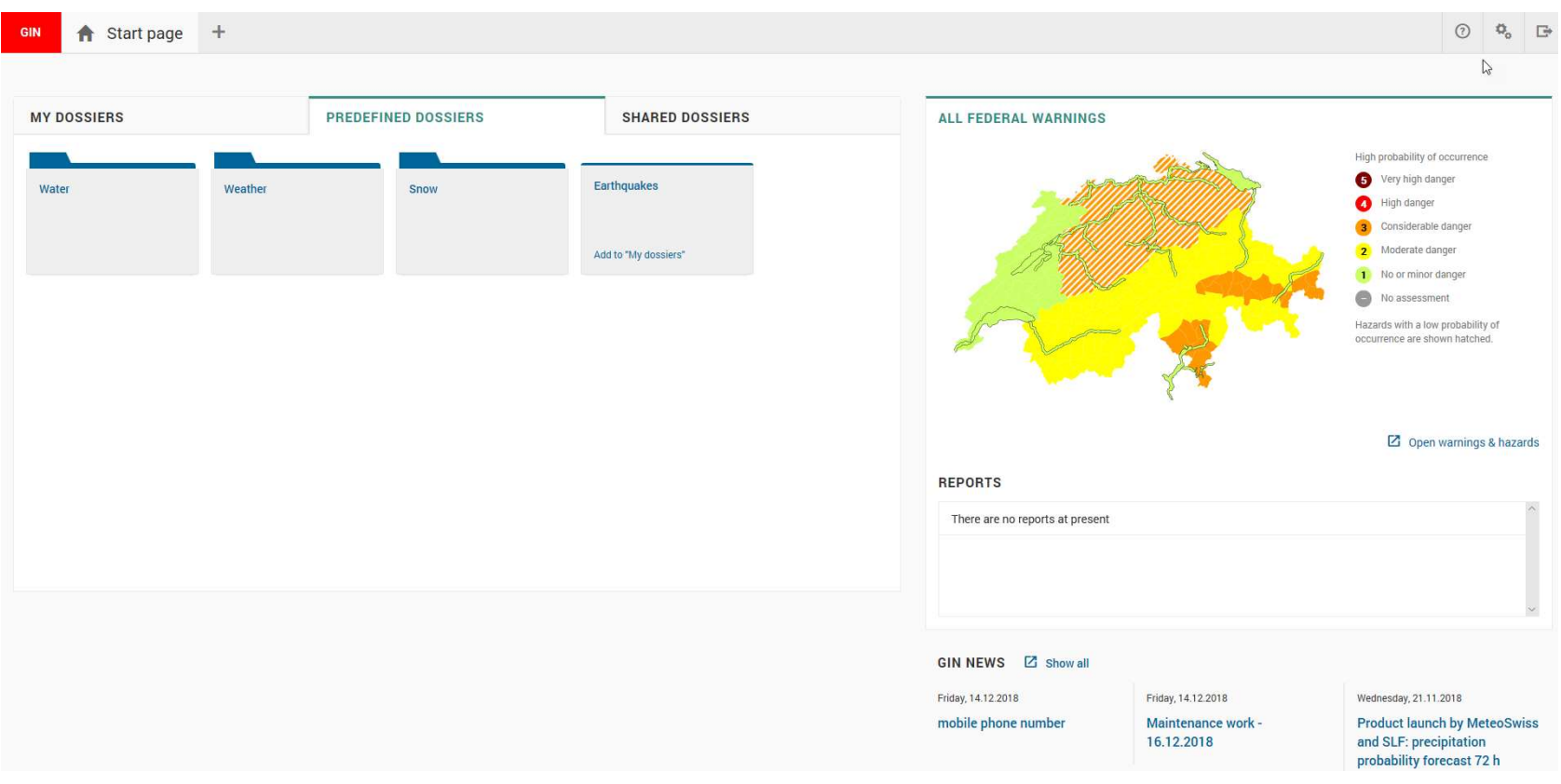

Figure 1. The new dashboard, designed to deliver an overview of the natural hazard situation in Switzerland.

In 2013 , an online survey showed that the platform does not fully meet user expectations, particularly as to user experience and usability of its cartographic, web-based user interface. Revaluation and redesign of the overall platform were necessary in order to improve map legibility, caused by the complexity of data, large data amounts, and high spatial density of online, real-time measurement data locations. A new web design and user interaction concept have been developed in 2014 and eventually put online in June 2017 . User acceptance testing by means of surveys and direct user feedback sessions were key factors in this perennial 
redesign process. The GIN platform now features important novel technical and graphical elements: The starting page is based on a dashboard containing virtual dossiers (Fig. 1), with which users configure their desired information, data, and map bundles individually, or use predefined adaptable views on various existing data sets. In addition, there is a new overall spatial search function to query data parameters. A responsive approach further improves the usability of the platform. The focus of these new features is on multi-views involving maps, diagrams, tables, text products, as well as selected geographical areas on maps, and fast data queries (Fig. 2). Current user feedback suggests that the new GIN platform design is well received, and that it is moving closer to its very goal: online monitoring and management of natural hazard events by enhanced usability, more targeted and higher personalization.

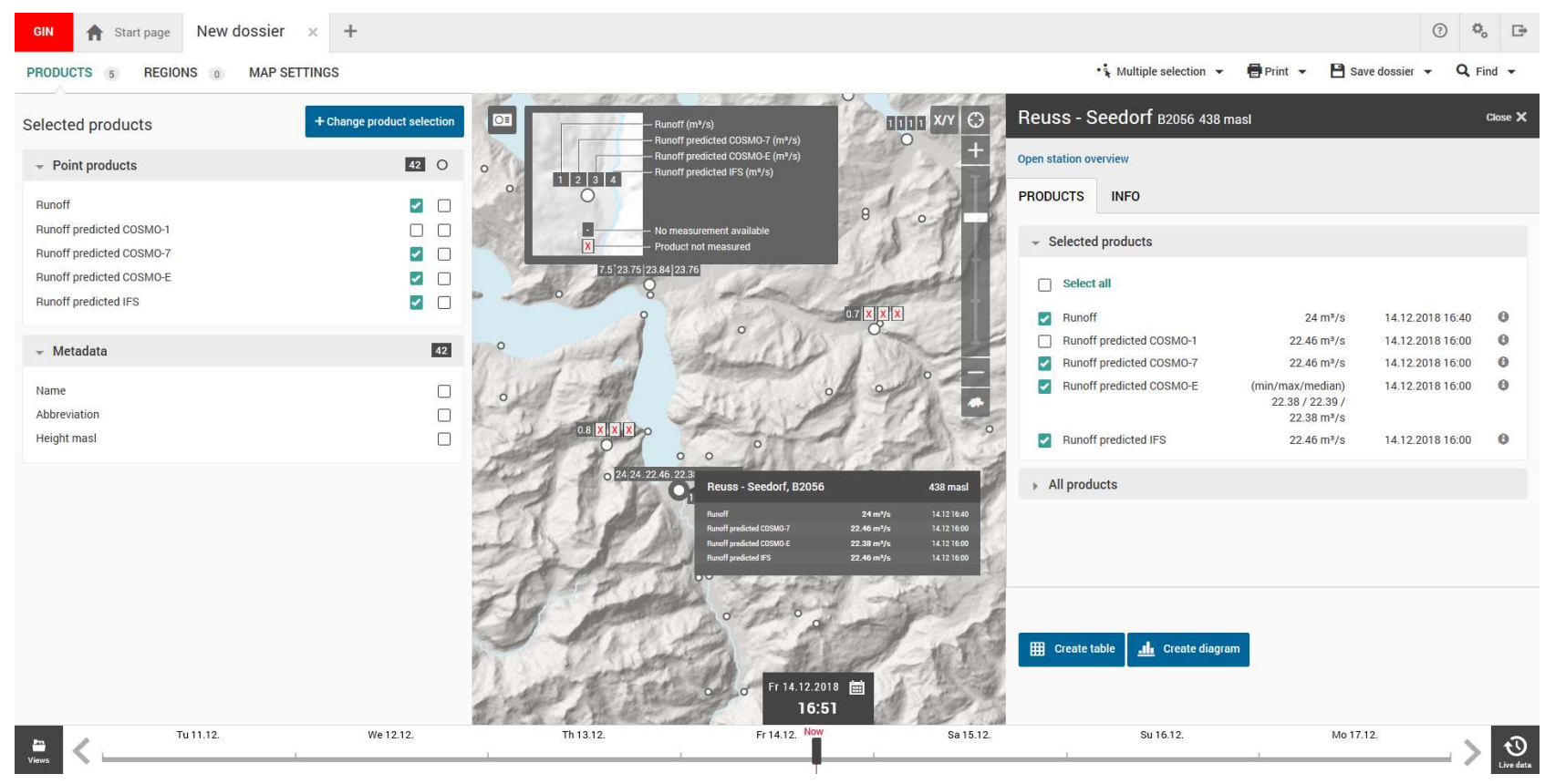

Figure 2. Multi-view of hydrological measuring stations, displaying forecasted river discharge.

Several Swiss Cantons (i.e., the political entities in Switzerland below the federation) actively participated, and still participate, in the conceptual GIN platform development process through advisory board meetings and consultations. On the operational level, Cantons actively provide and contribute further natural hazard information and measurement data from their own natural hazard monitoring networks. These additional Cantonal regional-scale data sets help to fill spatial data gaps, where no Federal data is available. GIN thusly integrates natural hazard data from Federal and Cantonal levels (and partly even private level), which adds value to all stakeholders on various political levels involved in natural hazard management (Federal, Cantonal, Regional, Communal crisis committees). Stakeholders not only use GIN's ample database and cartographic product portfolio to accomplish their early warning and crisis management tasks, but also benefit from seamless, secure and reliable IT-services, provided by the Swiss Federal Government. With the new GIN platform, Switzerland has a powerful, integrative, and comprehensive tool for monitoring and responding to natural hazard events. 